\title{
THE CHEMICAL AND ENZYMATIC HYDROLYSIS OF ARCHAEOLOGICAL WOOD CELLULOSE AND MONOSACCHARIDE PURIFICATION BY HIGH PH ANION EXCHANGE CHROMATOGRAPHY FOR COMPOUND-SPECIFIC RADIOCARBON DATING
}

\author{
G W L Hodgins ${ }^{1,2} \bullet$ T D Butters $^{3} \bullet$ C Bronk Ramsey ${ }^{1}$ R E M Hedges ${ }^{1}$
}

\begin{abstract}
Preliminary experiments were carried out on archaeological wood to investigate methods of cellulose hydrolysis and carbohydrate monomer purification for the purpose of compound-specific radiocarbon dating. The Chelford log, a known ${ }^{14} \mathrm{C}$ dead source of wood cellulose, was selected for study in order to investigate the levels of contamination introduced during sample purification. Two methods of hydrolysis were examined, mineral acid hydrolysis and enzyme hydrolysis using cellulase from Penicillium funiculosum. Under the conditions described, enzymolysis was far superior to acid hydrolysis in terms of the glucose monomer yield. Glucose monomer purification was accomplished using high $\mathrm{pH}$ anion exchange chromatography with pulsed amperometric detection. This high performance liquid chromatography (HPLC) method does not require sample derivatization and the chromatography products can be collected in water. These characteristics make it potentially well suited to carbon dating applications. ${ }^{14} \mathrm{C}$ dating of chromatographically purified glucose fractions revealed significant levels of contamination had accumulated during both protocols. Glucose contamination from the cellulase enzyme preparation was a major source of contamination within the enzymatically hydrolyzed samples. Ultrafiltration of the enzyme removed some but not all of this contamination. The contamination must be reduced 10-fold before the methodology could be viable for dating. This hydrolysis/HPLC method is also being investigated for ${ }^{14} \mathrm{C}$ dating of other carbohydrate polymers such as chitin.
\end{abstract}

\section{INTRODUCTION}

The large molecular size and insolubility of carbohydrate polymers such as cellulose and insect chitin make them difficult to define chemically with precision. Contaminants within these materials are difficult to identify, and pretreatment strategies are contingent upon the assumption that the putative contaminants can be made more soluble than the polymers. In contrast, simple sugars are amenable to stringent biochemical characterisation, and so a possible strategy for radiocarbon dating contaminated carbohydrate samples is polymer hydrolysis followed by monomer purification. But, such a strategy requires substantial sample manipulation and consequently carries an increased risk of introducing laboratory contamination.

This study examines the feasibility of a polymer hydrolysis/monomer purification approach and investigates two methods of polymer hydrolysis, mineral acid hydrolysis and enzymolysis. These two methods have been investigated in the context of ${ }^{14} \mathrm{C}$ dating another biopolymer: collagen protein. Acid hydrolysis of collagen and single amino acid (hydroxyproline) purification has been investigated by Stafford et al. (1982), and collagenase enzyme digestion and tripeptide purification methods were developed by Van Klinken and Hedges (1992). Schimmelmann and DeNiro (1983) investigated enzymatic hydrolysis of cellulose followed by dialysis to separate products and reactants. Their study was carried out on modern samples destined for carbon stable isotope ratio measurement.

Chemical and enzymatic hydrolysis methods offer different advantages. The principle advantage of acid hydrolysis is that it does not require the addition of exogenous carbon. The problems are that

\footnotetext{
${ }^{1}$ Oxford Radiocarbon Accelerator Unit, Research Laboratory for Archaeology and the History of Art, 6 Keble Road, Oxford, United Kingdom OX1 3QJ

${ }^{2}$ Corresponding author. Current address: CAIS, University of Georgia, 120 Riverbend Rd., Athens, Georgia 30602 USA. Email: ghodgins@arches.uga.edu.

${ }^{3}$ Glycobiology Institute, Department of Biochemistry, University of Oxford, South Parks Road, OX1 3QU
}

(C) 2001 by the Arizona Board of Regents on behalf of the University of Arizona Radiocarbon, Vol 43, Nr 2A, 2001, p 209-215

Proceedings of the 17 th International ${ }^{14} \mathrm{C}$ Conference, edited by I Carmi and E Boaretto 
the cleavage sites are randomly distributed so that the hydrolysis products are not necessarily monomers and, the acidic $\mathrm{pH}$ also promotes side reactions that can reduce the yield of monomer product, especially if contaminants such as amino acids are present. Furthermore, the neutralization of the acid $\mathrm{pH}$ after hydrolysis produces salts that can complicate sample recovery for ${ }^{14} \mathrm{C}$ measurement. Enzymolysis has the advantage that appropriately selected enzymes can favour the production of monomer product over oligomer and the potential for side reactions minimized. The down side of this approach is that enzymolysis contaminates the sample with modern organic materials, namely the enzyme, its contaminants, and its associated buffer salts. Therefore, the target monomer isolation protocol must be capable of rigorously excluding these diverse contaminants.

In an effort to purify carbohydrate monomers from polymer hydrolysates, this study investigates an HPLC method known as high pH anion exchange chromatography (HPAE) (Hardy and Townsend 1988; Hardy et al. 1988). The method is used widely in glycobiology for the analysis of carbohydrates. It has several characteristics that are potentially advantageous for compound-specific ${ }^{14} \mathrm{C}$ dating studies. The HPLC system and column matrix are designed to function in strong alkali and consequently are unusually stable. The method allows the separation and detection of underivatized carbohydrate molecules, and combined with continuous flow anion exchange membrane desalting, the chromatography products are recovered in water, facilitating direct combustion for ${ }^{14} \mathrm{C}$ measurement.

High $\mathrm{pH}$ anion exchange chromatography and online anion exchange membrane desalting have not been applied previously to the problems of compound specific ${ }^{14} \mathrm{C}$ dating. Consequently, the familiar suite of questions concerning column and detector capacity, the possible complications of sample cross contamination, sample contamination by solvent residues, column degeneration, and others, require empirical examination. Here we report on preliminary findings.

\section{MATERIALS AND METHODS}

The Chelford Log (ORAU sample no. P431, recovered by J Gowlett from the Early Devensian type site at Chelford, England) was used throughout this study. Previously, cellulose extracted from it has been dated to greater than 55,000 yr BP (OxA-124, Q-7). Cellulose was prepared using the standard ORAU acid/alkali/acid/chlorite bleach protocol (Hedges et al. 1989). Ultrafiltration was carried out using $0.5 \mathrm{~mL}$ polyethersulphone-based 10,000 nominal molecular weight limit (nmwl) UltrafreeMC Biomax-10 filters from Millipore Corp. These filters were pre-washed by repetitive centrifugation with deionized water before use. Cellulase enzyme (EC 3.2.1.4) type VII from Penicilium funiculosum, was obtained from Sigma-Aldrich Co. Ltd (cat no. C 0901). The specific activity was 5.9 units $/ \mathrm{mL}$. The enzyme was reconstituted at $10 \mathrm{mg}$ per milliliter in deionized water. Carbonate-free $50 \% \mathrm{NaOH}$ from Fisher Scientific was used to make the $200 \mathrm{mM} \mathrm{NaOH}$ eluate. It was prepared according to the Dionex protocol. Deionized water was Millipore MilliQ grade. All other chemicals were ACS grade.

\section{The HPLC System}

High $\mathrm{pH}$ anion exchange chromatography with pulsed amperometric detection was carried out on a system from Dionex Corp. (see Figure 1). The column, a Dionex Carbopac PA-1 (9 mm $\times 250 \mathrm{~mm}$ ) semi-preparative anion exchange column, was run isocratically, at room temperature, in carbonatefree $200 \mathrm{mM} \mathrm{NaOH}$ flowing at $1.7 \mathrm{~mL} / \mathrm{min}$. Carbohydrates were detected using a pulsed amperometric detector (PAD) cell (Dionex PAD-1). The device measures the ability of solutes to carry charge. A carbohydrate membrane desalter (Dionex CDM-1) was installed downstream of the pulsed amperometric detector to neutralize the $\mathrm{pH}$ and remove the resulting ions. Seventy-five mil- 


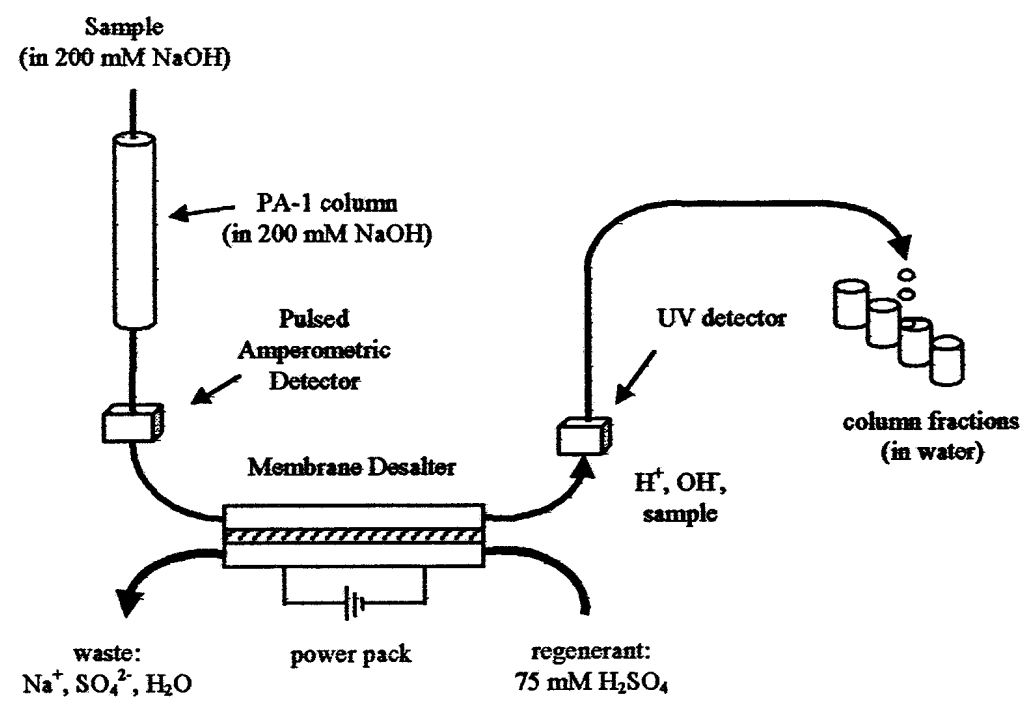

Figure 1 The schematic of the HPLC high $\mathrm{pH}$ anion exchange column, detectors, desalting system and fraction collector

limolar $\mathrm{H}_{2} \mathrm{SO}_{4}$ was used as the $\mathrm{CDM}-1$ chemical regenerant combined with electrolytic regeneration using a SRC-1 powerpack. A Variable Wavelength Detector (Dionex VWD) downstream of the membrane desalter and was used to detect molecules absorbing at $215 \mathrm{~nm}$. Comparison of the output from the two detectors provided a check on the performance of the membrane desalter. The volume between the detectors was approximately $350 \mu$ l. Fractions containing the desired peaks (approximately $2 \mathrm{~mL}$ each) were collected into glass tubes using a Pharmacia Frac-100 fraction collector, and then lyophylized.

\section{Acid Hydrolysis}

The acid hydrolysis protocol was adapted from the Schimmelmann and DeNiro (1986) protocol designed for the hydrolysis of chitin. Thirty milligrams of Chelford $\log \mathrm{A} / \mathrm{A} / \mathrm{A}$ cellulose were dissolved in $6.3 \mathrm{~N} \mathrm{HCl}$ at $10 \mathrm{mg} / \mathrm{mL}$ and placed at $95{ }^{\circ} \mathrm{C}$ for $1 \mathrm{hr}$. A supernatant was recovered after centrifugation for $5 \mathrm{~min}$ at $5000 \mathrm{G}$. The insoluble pellet was washed twice with water and the washes pooled with the supernatant. The supernatant was lyophylized and weighed. The lyophylisate was resuspended in water and ultrafiltered using the Biomax $10 \mathrm{~K} \mathrm{nmwl} \mathrm{centrifugal} \mathrm{filters.} \mathrm{The} \mathrm{less} \mathrm{than}$ $10,000 \mathrm{nmwl}$ fraction was recovered for high $\mathrm{pH}$ anion exchange chromatography.

\section{Enzyme Hydrolysis}

Ten milligrams of Chelford log A/A/A cellulose was mixed with 18 units $(3.0 \mathrm{mg})$ of $>10 \mathrm{~K} \mathrm{nmwl}$ filtered cellulase from P. funiculosum in $0.5 \mathrm{~mL}$ of Milli Q water. The enzyme and substrate were incubated at $37^{\circ} \mathrm{C}$ for $8 \mathrm{hr}$. The reaction tube was centrifuged at $5000 \mathrm{G}$ to pellet the undigested material and the supernatant recovered and ultrafiltered through a $10 \mathrm{kD} \mathrm{nmwl} \mathrm{membrane.} \mathrm{The} \mathrm{flow}$ through was purified by high $\mathrm{pH}$ anion exchange HPLC. The glucose-containing fractions were recovered and lyophylized in preparation for ${ }^{14} \mathrm{C}$ measurement. 


\section{${ }^{14} \mathrm{C}$ Measurement}

The lyophylized HPLC column fractions were transferred to the AMS system by resuspending each in $15 \mu \mathrm{l}$ of Milli Q water and pipetting the liquid to tin capsules containing Chromasorb W AW (Jones Chromatography Ltd, Hengoed, Mid Glamorgan, UK), a carbon-free diatomaceous binding agent. The sample-containing capsules were air-dried at room temperature for $30 \mathrm{~min}$, and then the capsules crimped closed in preparation for combustion.

Sample combustion and ${ }^{14} \mathrm{C}$ measurements were carried out using an online combustion system recently developed at ORAU (Ramsey and Humm, in press). The system is designed to cope with very small samples and was the logical choice for this initial study because of the apparently small size of the glucose-containing HPLC fraction derived from acid hydrolysis. The system currently does not allow measurement of carbon stable isotope values.

\section{RESULTS AND DISCUSSION}

Chromatography of glucose standard solutions established column capacity, detector responses, and the column and regenerant flow rates required for membrane desalter function. The input solvent $(200 \mathrm{mM} \mathrm{NaOH})$ emerged from the fraction collector with a $\mathrm{pH}$ of 4.8 to 5.0 and a conductivity less than that of a $50 \mu \mathrm{M} \mathrm{NaOH}$ standard solution. Glucose passed the PAD detector between 8.2 and 9.2 min post injection as a single peak. When the pulsed amperometric detector was set at maximum attenuation, $300 \mu \mathrm{g}$ of glucose per peak could be analyzed without detector saturation. The column capacity appeared to substantially exceed this quantity.

\section{Production and Analysis of Acid Hydrolysates}

Acid hydrolysis of $30 \mathrm{mg}$ of Chelford log cellulose by $\mathrm{HCl}$, in addition to breaking down the polymer, generated substantial quantities of insoluble material. The hydrolysate was ultrafiltered and only $1.6 \mathrm{mg}$ of material, representing a 5.3\% yield from the starting mass, passed through the 10,000 $\mathrm{nmwl}$ filter. When this was analyzed by high $\mathrm{pH}$ anion exchange chromatography a series of evenly spaced peaks within the PAD chromatogram suggested the presence of mono-, di-, tri- and oligosaccharides within the hydrolysate (Figure 2). The UV detector trace revealed a range of absorption peaks across the chromatogram. Many of these substances did not generate a PAD detector response.

The peak corresponding to monomeric glucose was collected, lyophylized, and combusted for AMS measurement. Only $42 \mu \mathrm{g}$ of carbon were recovered after combustion, and ${ }^{14} \mathrm{C}$ AMS measuremenrevealed $21.5 \% \pm 0.3 \%$ of this was modern carbon. At this stage the mode of contamination is not known.

\section{HPLC of $P$. funiculosum Cellulase Enzyme Solution}

Commercially available enzyme preparations even of high purity are often complex mixtures. Cellulase from P. funiculosum is not a single enzyme but an enzyme complex. It contains multiple activities, including both exo- and endo- $\beta$-glucosidic activities and is capable of degrading native cellulose to glucose (Wood et al. 1980; Wood and McCrae 1982; Sahasrabudhe et al. 1987). The published molecular weights of components within the complex range from 46 to $56 \mathrm{kD}$.

Before carrying out enzymatic digestion of the Chelford log cellulose, a sample of the Sigma $P$. funiculosum cellulase, prepared in Milli $\mathrm{Q}$ water, was analyzed by high $\mathrm{pH}$ anion exchange chromatography. A glucose-like peak with the characteristic high conductivity in the PAD detector and only weak absorption in UV detector, eluted from the PA-1 column at 8.2-9.0 min (Figure 3). In an effort 


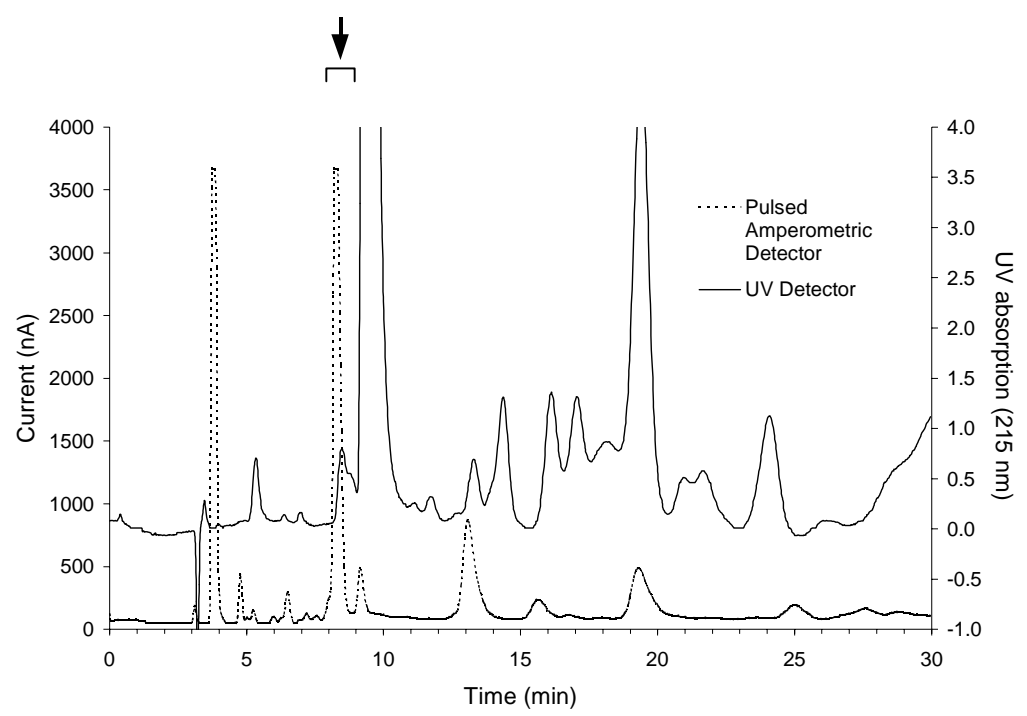

Figure 2 Chelford $\log$ acid hydrolysate $<10 \mathrm{~K}$ mw fraction. The putative glucose peak is marked.

to remove the contaminant, the enzyme preparation was ultrafiltered using a $10 \mathrm{kD} \mathrm{nmwl} \mathrm{mem-}$ brane. The retained fraction was recovered and $3 \mathrm{mg}$ analyzed by high $\mathrm{pH}$ anion exchange chromatography (Figure 4). The chromatogram revealed that the bulk of the contaminant was removed. Quantitation, based upon the chromatography of glucose standard solutions, indicated that $8 \mu \mathrm{g}$ of glucose contamination per milligram of ultrafiltered enzyme remained. The remaining traces were collected for combustion and ${ }^{14} \mathrm{C}$ measurement. Combustion and AMS measurement indicated the peak contained $15 \mu \mathrm{g}$ of carbon that was $144 \% \pm 7.4 \%$ modern. Enzyme digestions of the Chelford log cellulose were carried out using the ultrafiltered enzyme fraction.

\section{Production and Analysis of Enzyme-Catalyzed Cellulose Hydrolysates}

Three milligrams of the ultrafiltered enzyme was incubated with $10 \mathrm{mg}$ of Chelford log cellulose. After digestion, $3.1 \mathrm{mg}$ of soluble material passed through the $10 \mathrm{~K} \mathrm{nmwl}$ ultrafilter. This represented approximately a 30\% hydrolysis yield. Aliquots of this ultrafiltered hydrolysate were analyzed by chromatography on the Dionex PA-1 column (Figure 5) In contrast to the complexity of the acid hydrolysates, the enzyme hydrolysates contained predominantly a single component with an elution time similar to that of the glucose standard.

This peak was collected for ${ }^{14} \mathrm{C}$ dating. Combustion indicated that the peak contained $310 \mu \mathrm{g}$ of carbon. AMS indicated it was $5.8 \% \pm 0.3 \%$ modern. The majority of this modern carbon contamination can be explained by the modern glucose contamination introduced within the ultrafiltered enzyme.

Clearly investigating methods of removing glucose from the cellulase enzyme is a high priority. These initial reactions were carried out using extremely high enzyme to substrate ratios. It is possible that a substantial reduction in modern carbon contamination can be achieved by reducing this ratio, very likely without sacrificing monomer yield. 


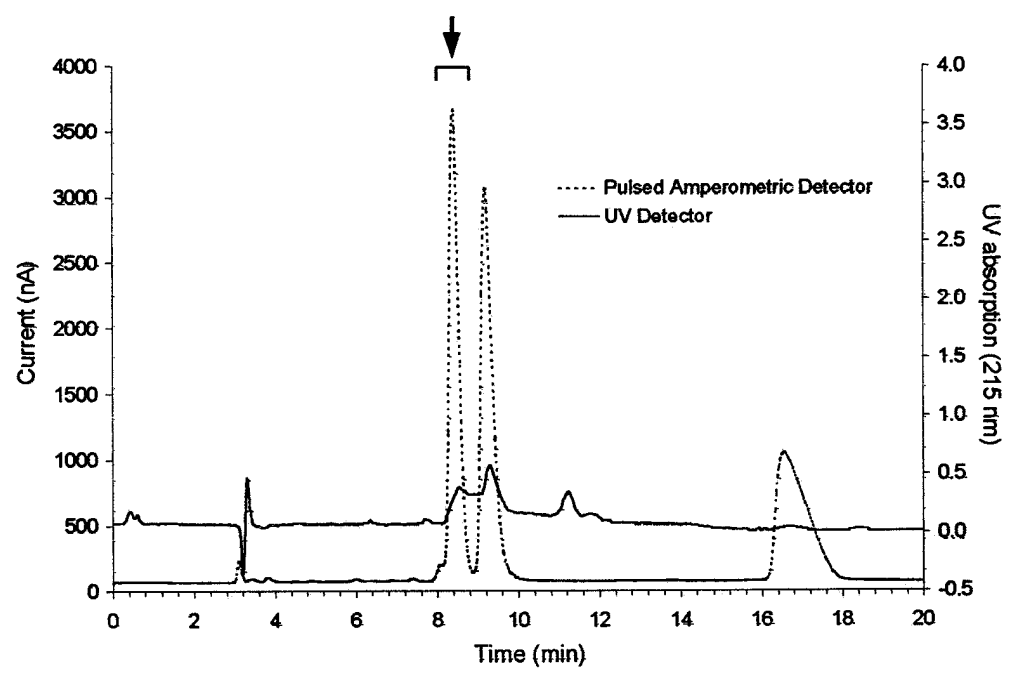

Figure 3 Cellulase from Penicillium funiculosum. Glucose standards eluted between 8.2 and $9.2 \mathrm{~min}$. The putative glucose peak within the enzyme fraction is marked with an arrow.

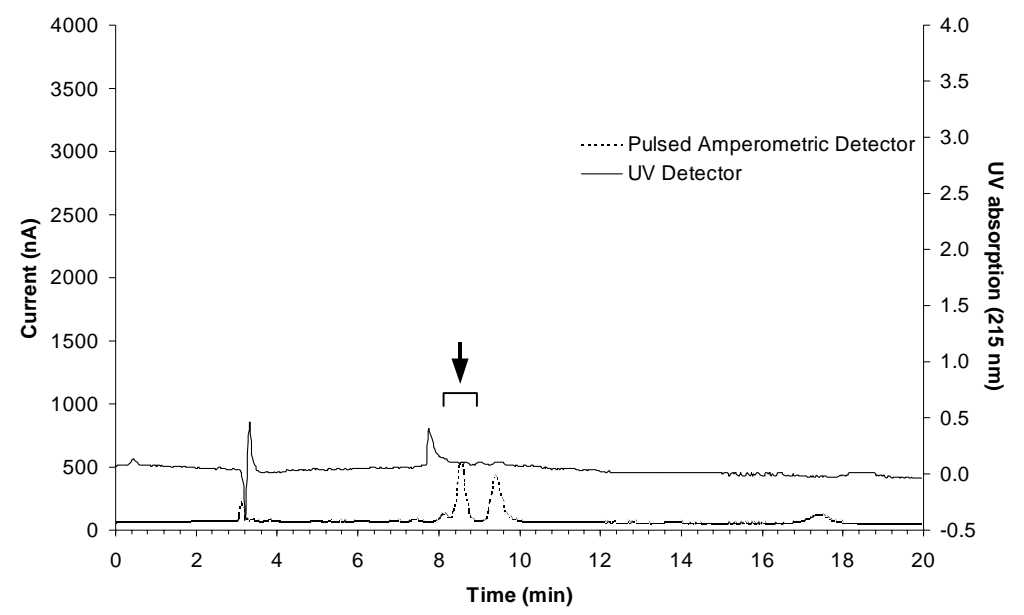

Figure 4 P. funiculosum cellulase $>10 \mathrm{k}$ mw fraction. The putative glucose peak, marked with the arrow, has been substantially reduced.

\section{CONCLUSION}

This study has provided a basic understanding of the potential and the challenges of compound-specific carbohydrate dating. A comparison of hydrolysis methods points towards future experiments that focus on enzymatic methods. Clearly these will be superior if glucose contamination within the enzyme preparation can be eliminated. An HPLC system has been designed with a capacity to handle samples large enough for subsequent stable isotope and ${ }^{14} \mathrm{C}$ measurements. The techniques of high $\mathrm{pH}$ anion exchange chromatography, pulsed amperometric detection, and anion exchange. 


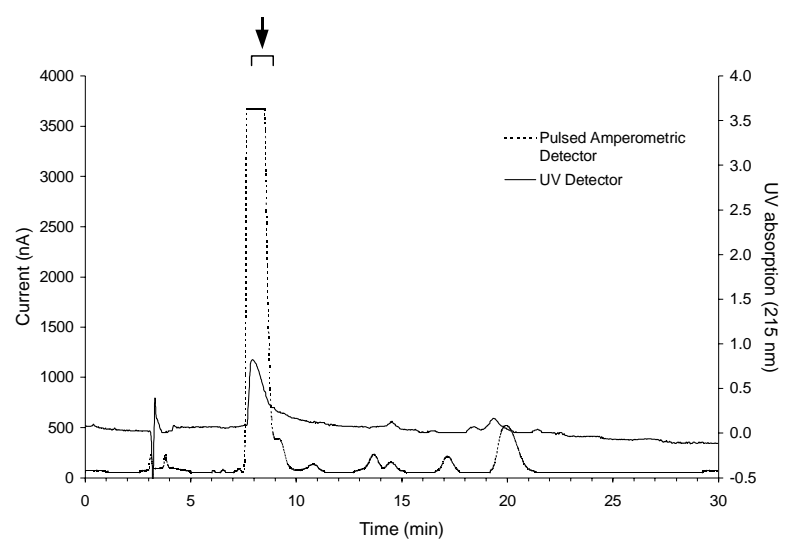

Figure 5 Chelford log Cellulase hydrolysate. Note that the putative glucose peak has eluted slightly before the elution time of the glucose standard marked with the arrow. It is possible that detector saturation has masked the existence of a double peak.

membrane desalting have shown potential and merit further investigation. Parallel studies on the hydrolysis and purification of sub-fossil insect chitin are ongoing.

\section{ACKNOWLEDGMENTS}

We gratefully acknowledge the assistance of Vanessa Pashley for preparing the Chelford Log A/A/ A cellulose fraction, and Ken Neal for suggesting the HPLC method.

\section{REFERENCES}

Hardy MR, Townsend RR. 1988. Separation of positional isomers of oligosaccharides and glycopeptides by high-performance anion-exchange chromatography with pulsed amperometric detection. Proceedings of the National Acadamy of Science USA 85:3289-93.

Hardy MR, Townsend RR, Lee YC. 1988. Monosaccharide analysis of glycoconjugates by anion exchange chromatography with pulsed amperometric detection. Analytical Biochemistry 170:54-62.

Hedges REM, Law I, Bronk CR, Housley RA. 1989. The Oxford accelerator mass spectrometry facility: technical developments in routine dating. Archaeometry 31(2):99-114.

Ramsey CB. Humm MJ. On-line combustion of samples for AMS and ionsource developments at ORAU. Proceedings of the 8th International Conference on Accelerator Mass Spectrometry, 6-10 September 1999, Vienna, Austria. In press.

Sahasrabudhe NA, Lachke AH, Ranjekar PK. 1987. Characterisation of the purified multifunctional cellulase component of Penicillium funiculosum. Biotechnology Letters 9(12):881-6.

Schimmelmann A, DeNiro MJ. 1983. Determination of carbon isotope ratios in plant starch via selective enzymatic hydrolysis. Analytical Chemistry 55:814-6.

Schimmelmann A, DeNiro MJ. 1986. Stable isotopic studies on chitin. I. Measurements on chitin/chitosan isolates and D-glucosamine hydrochloride from chitin. In: Muzzarelli RAA, Jeuniaux C, Gooday GW, editors. Chitin in nature and technology. New York: Plenum. p 357-64.

Stafford TW, Duhamel RC, Haynes CV Jr, Brendel K. 1982. Isolation of proline and hydroxyproline from fossil bone. Life Sciences 31:931-8.

van Klinken GJ, Hedges REM. 1992. Experiments on ${ }^{14} \mathrm{C}$ dating of contaminated bone using peptides resulting from enzymatic cleavage of collagen. Radiocarbon 34(3):292-5.

Wood TM, McCrae SI, MacFarlane CC. 1980. The isolation, purification and properties of the cellobiohydrolase component of Penicillium funiculosum cellulase. Biochemical Journal 189:51-6.

Wood TM, McCrae SI. 1982. Purification and some properties of a $(1 \rightarrow 4)-\beta$-D-glucan glucohydrolase associated with the cellulase from the fungus Penicillium funiculosum. Carbohydrate Research 110:291-303. 